\title{
Intermittent exotropia evaluation using the propedeutics proposed by Kushner: a 25-year study
}

\author{
Avaliação de exotropia intermitente usando propedêutica proposta \\ por Kushner: estudo de 25 anos \\ Ana Luiza Fontes de Azevedo Costa ${ }^{1,2}$, Thiago Gonçalves dos Santos Martins², lara Debert', Mariza Polati \\ 1. Universidade de São Paulo, São Paulo, SP, Brazil. \\ 2. Universidade Federal de São Paulo, São Paulo, SP, Brazil.
}

\begin{abstract}
Purpose: To evaluate the clinical and surgical impacts of phenomena that could occur in intermittent exotropia. Methods: The medical records of intermittent exotropia cases from 1991 to 2014 were retrospectively reviewed. All patients underwent a series of measures, including a protocol to assess monocular occlusion based on the propedeutics proposed by Kushner. Results: Outdoor sensitivity was observed in $31 \%$ of patients with an undercorrection rate of $44 \%$ vs. $18 \%$ of cases with no outdoor sensitivity. After $1 \mathrm{~h}$ of monocular occlusion, $41 \%$ of all patients achieved an increase in deviation with an undercorrection rate of $40 \%$, whereas $25 \%$ did not. Conclusion: The results show the importance of complete propedeutics, since there is a higher rate of late undercorrection in cases with outdoor sensitivity and increased deviation after occlusion.
\end{abstract}

Keywords: Exotropia; Strabismus; Oculomotor muscles; Sensory deprivation; Vision, monocular

RESUMO | Objetivo: Avaliar os impactos clínico e cirúrgico dos fenômenos que podem ocorrer na exotropia intermitente. Métodos: Os prontuários de casos de exotropia intermitente de 1991 a 2014 foram revisados retrospectivamente. Todos os pacientes foram submetidos a uma serie de medidas incluindo o protocolo com oclusão monocular com base na propedêutica proposta por Kushner. Resultados: Outdoor sensitivity foi observada em $31 \%$ dos pacientes com taxa de subcorreção de $44 \%$ vs. $18 \%$ dos casos sem outdoor sensitivity. Após 1 hora de oclusão monocular, $41 \%$ de todos os pacientes apresentaram

Submitted for publication: April 7, 2017

Accepted for publication: July 24, 2018

Funding: No specific financial support was available for this study.

Disclosure of potential conflicts of interest: The authors have no potential conflicts of interest to disclose.

Corresponding author: Ana Luiza Costa.

Av. Dr. Enéas de Carvalho Aguiar, 255 - São Paulo, SP - 05403-000 - Brazil E-mail: luizafacosta@hotmail.com

Approved by the following research ethics committee: Hospital das Clínicas da Faculdade de Medicina da Universidade de São Paulo (\# 1.957.370). um aumento no desvio com uma taxa de subcorreção $40 \%$, enquanto 25\% não. Conclusão: Os resultados demonstram a importância da propedêutica completa, uma vez que há maior taxa de subcorreção tardia nos casos de outdoor sensitivity e maior desvio após a oclusão.

Descritores: Exotropia; Estrabismo; Músculos oculomotores; Privação sensorial; Visão monocular

\section{INTRODUCTION}

The treatment of intermittent exotropia, or $\mathrm{X}(\mathrm{T})$, remains challenging because the degree of deviation varies according to the distance of fixation and is also influenced by factors related to fusional and accommodative vergences. Overcorrection occurs when the surgical plan aims to correct the greatest measured deviation. On the other hand, undercorrection with subsequent recurrence of deviation is frequent when the surgical plan is based on any measure other than the maximum value $^{(1)}$.

Integrated mechanisms responsible for the variability in deviation have been described and should be investigated in order to increase the chance of surgical success $^{(1)}$. To achieve satisfactory results of individualized treatment, whether clinical or surgical, complementary propedeutic tests are recommended to clarify the influence of fusional and accommodation components in the deviation and to classify the type of $X(T)$.

Scobee ${ }^{(2)}$ recommended monocular occlusion for $1 \mathrm{~h}$ to reveal the true near deviation while eliminating tenacious proximal fusion (TPF). The ratio of accommodative convergence to accommodation (AC/A) must, therefore, be obtained from measurements after occlusion $^{(3)}$. Kushner and Morton ${ }^{(4)}$ updated the classification of $\mathrm{X}(\mathrm{T})$ proposed by Burian, based on the understanding that these phenomena are related to the deviation. They 
described propedeutics that include measures at 6 and $0.3 \mathrm{~m}$. After occlusion for $1 \mathrm{~h}$ of the eye that deviates more frequently, measurement should be made while the patient fixates on a distant target outdoors, then repeated at 6 and $0.3 \mathrm{~m}$ without +3.0-diopter lenses (to assess the presence of TPF), then again with +3.0-diopter lenses (to exclude the accommodative component) and at $6 \mathrm{~m}$ with -1.50 or -2.00 diopter lenses added to the refraction (to stimulate accommodation). Finally, the $\mathrm{AC} / \mathrm{A}$ ratio is calculated using the gradient or heterophoria method.

$\mathrm{X}(\mathrm{T})$ is subdivided into the following three types: basic, when the distance/near difference is not more than 10 prism diopters (PD); convergence insufficiency when the near deviation is at least 10 PD greater than that of the distance; and pseudo-divergence excess (pseudo-high $\mathrm{AC} / \mathrm{A}$ ratio) when the distance deviation is at least $10 \mathrm{PD}$ higher than the near deviation prior to occlusion with an increase in near deviation after occlusion or after the measurement with the +3.0-diopter lenses. In true divergence excess, a distance deviation of at least 10 PD higher than the near deviation is observed, with no increase in near deviation after occlusion or with positive lens.

Distance and near measures are considered alike when the difference is within $10 \mathrm{PD}^{(4)}$. Cases with a high $\mathrm{AC} / \mathrm{A}$ ratio, which become normal after occlusion, are classified as pseudo-high AC/A. The normal range of the AC/A ratio would be 3-5:1.

Here, we report the results of a series of patients examined with this work-up in order to evaluate the clinical and surgical impacts of the phenomena that could occur in $\mathrm{X}(\mathrm{T})$.

\section{METHODS}

All records of patients with $\mathrm{X}(\mathrm{T})$ followed in the Ophthalmic Strabismus Sector of the Hospital das Clínicas, FMUSP, between 1991 and 2014 and with full examination results, according to the work-up proposed by Kushner and Morton ${ }^{(4)}$, were retrospectively reviewed. Only motor function was considered for a diagnosis of $\mathrm{X}(\mathrm{T})$. Sensorial examination results were not taken into consideration.

The following factors were evaluated: age at onset of deviation, age when treatment started, age at first surgery, sex, best corrected visual acuity, as measured using the Lea Hyvärinen table, refractive error under cycloplegic refraction with $1 \%$ cyclopentolate, distance and near measures preoperatively and at 6 and 12 months after surgery, family history of strabismus, follow-up period, and type of surgery performed.

In addition to the usual measurements, we routinely carry out a specific and standardized examination based on the work-up described by Kushner ${ }^{(1,4,5)}$. The monocular occlusion of the most commonly deviated eye for at least $1 \mathrm{~h}$ before the examination is required. The protocol includes a measurement with the patient fixating on a distant target outdoors through the office window, thereby submitting the patient to the outdoor sensitivity effect, which increases deviation and is caused by intense lighting and/or increased distance of the fixation point ${ }^{(1)}$. In this study, this phenomenon was considered to have occurred when the increase was $\geq 10$ PD. Then, we measured, in the office, for the distant target $(6 \mathrm{~m})$ and near targets $(0.3$ and $0.2 \mathrm{~m})$, and next with +3.00-diopter lenses for the $0.3 \mathrm{~m}$ target, in order to relax all accommodations, and finally with -2.0-diopter lenses to stimulate accommodation (miosis-accommodation-convergence synkinesis). During the entire examination, the patient was not allowed to regain binocularity, which was accomplished by keeping one eye always occluded. Near deviation was not measured without the +3.00 lenses previous to the $1 \mathrm{~h}$ monocular occlusion.

The AC/A ratio was calculated using the gradient method where the difference between the near measures with and without the +3.0 lenses after the monocular occlusion was divided by 3. An AC/A ratio of 2.5-6:1 was considered normal. The AC/A ratio was also calculated using the heterophoria method where the distance and near difference were divided by 3 and finally added to the interpupillary distance in centimeters, with the normal value at 3-9:1. When the AC/A ratio is high, a decrease in near deviation occurs and there is an increase in distant deviation when stimulated with -2.0 lenses. Another factor that decreases the near deviation is the TPF, which is eliminated after monocular occlusion for $1 \mathrm{~h}$.

The distance and near measures alike were considered when the difference did not exceed $10 \mathrm{PD}$. The diagnosis of pseudodivergence excess was based on the measures after monocular occlusion. An AC/A ratio within the normal range without TPF was classified as the basic type.

\section{RESULTS}

The study cohort consisted of 109 patients, of which $63 \%$ were female. The age at the onset of deviation varied from birth to 39 years, with $63 \%$ being in the first year of life. The age at which treatment started ranged 
from 1 to 49 years. There was a family history of strabismus in $52 \%$ of cases.

The best corrected visual acuity was 1.0 in both eyes in 82 (75\%) patients. Two patients had low vision, one with high myopia, and the other with nystagmus. Amblyopia, defined as a difference of at least two lines between the best corrected visual acuity of each eye, was observed in seven (6\%) patients. Considering the refractive error under cycloplegia, hyperopia was present in 19 patients, myopia in 3, hyperopic astigmatism in 31 , myopic astigmatism in 37 , and mixed astigmatism in 8. Four patients had antimetropia, and seven had no refractive error.

According to the classification, 26 (24\%) patients presented with pseudodivergence excess, 1 (1\%) with true divergence excess, 8 (7\%) with convergence insufficiency, and 74 (68\%) with the basic type.

The mean deviation, the occurrence of outdoor sensitivity, and the increase in deviation after occlusion for each patient are shown in table 1. As indicated, there was an increase in deviation after occlusion in all cases of pseudodivergence excess, but this increase did not reach 10 PD, although the underlying reasons are not described.

Surgery was suggested to 53 patients, of whom 41 agreed and 12 did not. Of the patients who underwent surgery, 13 had pseudodivergence excess, 1 had convergence insufficiency, and 27 had the basic type. The surgery was determined by the greatest obtained angle measurement. Reoperation was proposed to eight patients, five of whom agreed and three did not.

The mean age at the first surgery was 18.5 (range, 4-49) years. The pre- and postoperative measures are presented in table 2 . In the only case of the convergence insufficiency type, the patient was followed up for only 5 months postoperatively and had no distance or near deviation.

Of the patients with the basic type of $\mathrm{X}(\mathrm{T})$ with outdoor sensitivity, three were overcorrected, six were undercorrected, and surgery was successful in four, that is, $\mathrm{X}(\mathrm{T})$ of up to $10 \mathrm{PD}$. Of the pseudodivergence excess patients that underwent surgery, one was overcorrected, one was undercorrected, and one did not complete the 1 year follow-up.

At the 1 year postoperative follow-up, the mean deviation among the patients with the pseudodivergence excess type, residual $\mathrm{X}(\mathrm{T})$ was 9.5 and 8 PD for the distance and near measures, respectively. In the basic type, the mean deviation of residual $\mathrm{X}(\mathrm{T})$ was $8 \mathrm{PD}$ for the distance measure and 10 PD for the near. Four patients did not complete the 1 year follow-up, one patient had

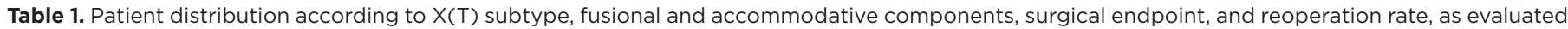
with a standardized examination over a 25 year period

\begin{tabular}{|c|c|c|c|c|c|}
\hline & $\begin{array}{c}\mathrm{N} \text { total/ } \\
\text { operated }\end{array}$ & Reoperation & $\begin{array}{c}\text { Outdoor sensitivity/ } \\
\text { mean }\end{array}$ & $\begin{array}{l}\text { Increase of deviation } \\
\text { after occlusion (\%) }\end{array}$ & $\begin{array}{c}\text { High AC/A } \\
\text { ( } n \text { of patients) }\end{array}$ \\
\hline Pseudo-excess of divergence (pseudo-high AC/A) & $26 / 13$ & 1 & $n=10 / 14.7 P D$ & $18(69 \%)$ & 4 \\
\hline Convergence insufficiency & $8 / 1$ & 0 & $n=1 / 22 P D$ & $4(50 \%)$ & 0 \\
\hline Basic type & $74 / 27$ & 4 & $n=23 / 15.5 P D$ & $23(31 \%)$ & 3 \\
\hline True divergence excess & $1 / 0$ & 0 & $\mathrm{n}=0$ & 0 & 0 \\
\hline Total & $109 / 41$ & 5 & $\mathrm{n}=34(31 \%)$ & $\mathrm{n}=45(41 \%)$ & 7 \\
\hline
\end{tabular}

$\mathrm{N}=$ number; $\mathrm{PD}=$ prismatic diopters.

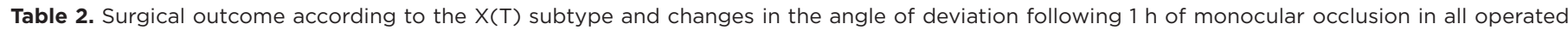
cases, as evaluated with a standardized examination over a 25 year period.

\begin{tabular}{|c|c|c|c|c|}
\hline & $\begin{array}{l}\text { Mean initial distance deviation/ } \\
\text { near (before occlusion) }\end{array}$ & $\begin{array}{c}\text { Mean preoperative distance } \\
\text { deviation/near (before } \\
\text { occlusion//after occlusion) }\end{array}$ & $\begin{array}{c}\text { Mean } 6 \text { months } \\
\text { postoperative distance } \\
\text { deviation/near }\end{array}$ & $\begin{array}{c}\text { Mean } 1 \text { year } \\
\text { postoperative distance } \\
\text { deviation; near }\end{array}$ \\
\hline $\begin{array}{l}\text { Pseudo-excess of divergence } \\
\text { (pseudo-high } \mathrm{AC} / \mathrm{A} \text { ) }\end{array}$ & $31 / 15$ PD & 31/16// 30/29 PD & $\begin{array}{c}\text { 2/3.2 PD } \\
\text { (1 overcorrections, } 0 \\
\text { undercorrections) }\end{array}$ & $\begin{array}{c}\text { 9.5/8 PD } \\
\text { (1 overcorrections, } \\
4 \text { undercorrections) }\end{array}$ \\
\hline Convergence insufficiency & 25/31 PD & 70/80 // 75/85 PD & - & - \\
\hline Basic type & $27 / 27 \mathrm{PD}$ & 35/36 // 36/39 PD & $\begin{array}{c}\text { 7.1/7.8 PD } \\
\text { (3 overcorrections, } \\
4 \text { undercorrections) }\end{array}$ & $\begin{array}{c}\text { 8/10 PD } \\
\text { (3 overcorrections, } \\
10 \text { undercorrections) }\end{array}$ \\
\hline True divergence excess & 20/4 PD & - & - & - \\
\hline
\end{tabular}


esotropia (ET) for the distance and near measures, and another had ET only for the distance. Four patients with the pseudodivergence excess type did not complete the 1 year postoperative follow-up, and one patient had ET for the distance and near measures.

Among the pseudodivergence excess cases, five surgeries were lateral rectus recession and medial rectus resection, and eight were bilateral lateral recession. In the case of convergence insufficiency, the surgery was recession and resection. For the basic type, 22 were recession and resection, 4 were bilateral lateral recession, and 1 was lateral rectus recession.

\section{DISCUSSION}

The intermittence of $X(T)$ is due to failures in at least one of the following four main mechanisms: fusional convergence, accommodative convergence, proximal convergence, and tonic convergence. The purpose of the monocular occlusion for $1 \mathrm{~h}$ is to neutralize the fusional component in order to decompensate the entire deviation and the TPF while not allowing the patient to recover binocularity during the examination. The proposed protocol also enables the calculation of the AC/A ratio, which can be obtained by the gradient method or the heterophoria method. As for cases with a high AC/A ratio, it is usually supposed that there will be a decrease in the near deviation and the distance measure with a -2.0-diopter lens. Without occlusion, it is not possible to define the cause of a decrease in near deviation.

The phenomena of outdoor sensitivity and increased deviation after $1 \mathrm{~h}$ of monocular occlusion were not limited to patients with a distance deviation greater than the near one, thereby justifying the use of complete propedeutics in any patient with $\mathrm{X}(\mathrm{T})$.

In previous studies, the undercorrection rate was $40 \%$ among patients with outdoor sensitivity whose surgery was planned on the basis of the $6 \mathrm{~m}$ measures. Patients who showed increases in measures after $1 \mathrm{~h}$ of monocular occlusion, but who had surgery planned for the measures obtained before occlusion, had a $35 \%$ undercorrection rate. In contrast, the undercorrection rate was reported to be $18 \%$ for a group with no outdoor sensitivity or increased deviation after occlusion ${ }^{(1)}$.

In this study, the outdoor sensitivity phenomenon was observed in $31 \%$ of cases, whereas the undercorrection rate was $44 \%$ and $18 \%$ in the group that did not have outdoor sensitivity. The group that had the greatest number of cases with outdoor sensitivity was the pseudodivergence excess type. Four patients in this group showed an increase in deviation that was only caused by outdoor sensitivity. After $1 \mathrm{~h}$ of monocular occlusion, $41 \%$ of the cases showed an increase in deviation. The undercorrection rate in these cases was $40 \%$ and $25 \%$ in the group with no increase in deviation after occlusion. Despite the increase in the surgical dose, the rate of undercorrection was higher. Perhaps, a greater increase in the surgical dose is needed to avoid late undercorrection.

The only case of true divergence excess had a normal AC/A ratio. Kushner and Morton ${ }^{(4)}$ described divergence excess as an excess of proximal convergence, which may be due to accommodative convergence or proximal accommodation. Patients with a normal AC/A ratio have excess proximal convergence. However, TPF and proximal convergence should not be confused with one other. In fact, TPF is the delay in dissipating the proximal convergence, which can be eliminated by prolonged occlusion or the adaptation of prisms, whereas proximal convergence does not dissipate with prolonged occlusion or prisms and is probably related to the awareness that something is close to the eyes.

Kushner proposes surgical planning for the correction of the greatest angle of deviation measured for distance and infinity, arguing that undercorrection often occurs in cases where the deviation in the infinity measure is greater than the deviation of the $6 \mathrm{~m}$ measure ${ }^{(1)}$.

As described by Kushner, most of the patients in the present study were female. However, other studies have reported a male prevalence $\mathrm{e}^{(7,8)}$.

The majority of the cases in Kushner's study ${ }^{(4)}$ had the pseudo-excess of divergence type, whereas most of the cases in the present study had the basic type, as demonstrated in other studies ${ }^{(7,9)}$. TPF was found in all patients with pseudo-excess of divergence, in agreement with the results of the referred author. However, the true excess divergence type is unusual when the measure is made in accordance with the $\operatorname{protocol}^{(1,10)}$.

Many cases of pseudo-excess of divergence are due to the presence of TPF ${ }^{(1)}$. Measurements prior to occlusion demonstrate increased distance deviation and decreased near deviation.

When there is a significant fusional component, which changes with the occlusion, the surgical result is usually satisfactory because of the presence of binocularity, which would promote self-adjustment of the deviation.

Overcorrection can be avoided, if an elevation in the $\mathrm{AC} / \mathrm{A}$ ratio is identified in the preoperative period. In this study, there was overcorrection in one single case of the pseudo-excess of divergence group that had outdoor 
sensitivity and a normal AC/A ratio, but with TPF. There were also three cases of overcorrection in patients with basic type $\mathrm{X}(\mathrm{T})$ and no increase in the $\mathrm{AC} / \mathrm{A}$ ratio, but had outdoor sensitivity, suggesting that the surgical dose in these cases was increased beyond what was necessary.

The indicated surgery was based on the classification that was determined in accordance with the results of the proposed protocol. Thus, basic type $\mathrm{X}(\mathrm{T})$ and the convergence insufficiency type were corrected by monocular recession and resection. In the pseudo-excess of divergence type, monocular recession and resection or bilateral lateral recession were performed.

When the AC/A ratio is high, such as in $60 \%$ of patients with true excess of divergence and many patients with pseudo-excess of divergence, one should be careful with recession/resection surgery because of the risk of overcorrection, according to one study(6).

There were two main limitations to this study that should be considered. First, the prism adaptation test was not part of the described examination sequence, which likely would have enhanced the deviation in selected cases and provided additional information. Second, the results are based on measurements obtained during a 1 year postoperative follow-up period, which could be considered rather short, especially if the rate of late recurrence of $\mathrm{X}(\mathrm{T})$ is taken into account.

In this retrospective investigation, we attempted to clarify the principles involved in carrying out $\mathrm{X}(\mathrm{T})$ and the strategies that should be followed in order to increase treatment success rates. Future prospective studies are warranted to further investigate the impact of the use of this detailed propedeutic, as compared with traditional propedeutics, over a long-term follow-up period.

\section{REFERENCES}

1. Kushner BJ. The distance angle to target in surgery for intermittent exotropia. Arch Ophthalmol. 1998;116(2):189-94. Comment in: Arch Ophthalmol. 1998;116(11):1550.

2. Scobee RG. Exophoria. In: The oculo-rotary muscles. St Louis: CV Mosby; 1952. p.59-61.

3. Cooper J, Medow N. Intermittent exotropia basic and divergence excess type. Binocul Vis Eye Muscle Surg Q. 1993;8(3):185-216.

4. Kushner BJ, Morton GV. Distance/near differences in intermittent exotropia. Arch Ophthalmol. 1998;116(4):478-86.

5. Kushner BJ. Selective surgery for intermittent exotropia based on distance/near differences. Arch Ophthalmol. 1998;116(3):324-8.

6. Kushner BJ. Exotropic deviations: a functional classification and approach to treatment. Am Orthopt J. 1988;38(1):81-93.

7. Jung JW, Lee SY. A comparison of the clinical characteristics of intermittent exotropia in children and adults. Korean J Ophthalmol. 2010;24(2):96-100.

8. Wang B, Wang L, Wang Q, Ren M. Comparison of different surgery procedures for convergence insufficiency-type intermittent exotropia in children. Br J Ophthalmol. 2014;98(10):1409-13.

9. Walklate KJ. The role of diagnostic occlusion in distance exotropia. Br Orthopt J. 1998;55:2-7.

10. Yang M, Chen J, Shen T, Kang Y, Deng D, Lin X, et al. Clinical characteristics and surgical outcomes in patients with intermittent exotropia: a large sample study in south China. Medicine (Baltimore). 2016;95(5):e2590. 